\title{
Classification of Fermi-LAT sources with deep learning
}

\author{
Silvia Manconi, ${ }^{a, *}$ Anja Butter, ${ }^{b}$ Thorben Finke, ${ }^{a}$ Felicitas Keil ${ }^{a}$ and Michael Krämer ${ }^{a}$
}

${ }^{a}$ Institut für Theoretische Teilchenphysik und Kosmologie, RWTH Aachen University, Germany

${ }^{b}$ Institut für Theoretische Physik, Heidelberg University, Germany

E-mail: manconi@physik.rwth-aachen.de

Machine learning techniques are powerful tools for the classification of unidentified gamma-ray sources. We present a new approach based on dense and recurrent deep neural networks to classify unidentified or unassociated gamma-ray sources in the last release of the Fermi-LAT catalog (4FGL-DR2). Our method uses the actual measurements of the photon energy spectrum and time series as input for the classification, instead of specific, hand-crafted features. We focus on different classification tasks: the separation between extragalactic sources, i.e. Active Galactic Nuclei (AGN), and Galactic pulsars, the further classification of pulsars into young and millisecond pulsars and the sub-classification of AGN into different types. Since our method is very flexible, we generalise it to account for uncertainties in the predicted classes. Our list of high-confidence candidate sources labelled by the neural networks provides targets for further multiwavelength observations to identify their nature, as well as for population studies, and is available at https: //github.com/manconi/agn-psr-nn-classification.

$37^{\text {th }}$ International Cosmic Ray Conference (ICRC 2021)

July 12th - 23rd, 2021

Online - Berlin, Germany

\footnotetext{
*Presenter
} 


\section{Introduction}

The Fermi Large Area Telescope (LAT) is a pair-conversion telescope which surveys the entire sky, detecting gamma-rays with energies from few tens of $\mathrm{MeV}$ up to the $\mathrm{TeV}$ range. Observed sources are distinguished according to their position and spectral characteristics, and have been collected in a succession of public source catalogs. The fourth Fermi-LAT gamma-ray catalog, Data Release 2 (4FGL-DR2) [1, 2] is the latest version, and it is based on the first ten years of data taking with gamma-ray energies ranging from $50 \mathrm{MeV}$ to $1 \mathrm{TeV}$. An overview of the composition of the 4FGL-DR2 is reported in Fig.1, where boxes with continous lines summarize the source class statistics as reported in the catalog. The most numerous and brightest source population is currently represented by Active Galactic Nuclei (AGN), where jets originating from a supermassive black hole at the centre of a galaxy emit electromagnetic radiation in a broad range [3]. If the jet is pointing towards the line of sight, AGN are called blazars. The vast majority (more than $90 \%$ ) of detected AGN are blazars. Blazars are further classified into BL Lacs (BLL) or Flat Spectrum Radio Quasars (FSRQ) objects according to optical spectral characteristics, the latter showing strong, broad emission lines [1,2]. About one third of detected blazar is still classified to be of uncertain type (BCU). Aside from AGN, the second main class of sources contained in present catalogs consists of gamma-ray pulsars (PSR), divided further into young pulsars (YNG) and millisecond pulsars (MSP), the last believed to be old pulsars for which the period has decreased via accretion from a companion object [4]. The AGN and PSR classes of gamma-ray emitters are characterised by different timing and spectral properties. While AGN typically have a variable gamma-ray flaring pattern on time scales of months, PSR are instead non-variable on long time scales. Moreover, the energy spectra of AGN are generically well described by a power law shape, which is softer than that of a PSR. The energy spectra of PSR have a spectral curvature, where a break is observed at low and high energies in the Fermi-LAT energy band, requiring the addition of an exponential cutoff at a few GeV. In the 4FGL-DR2 catalog the gamma-ray sources are identified as, or associated with, an AGN, a PSR, or some more rare population class. Sources are identified according to a correlated timing signature at different wavelengths, or only associated requiring positional coincidence with a counterpart source at other wavelengths. A total of 1794 sources (about one third) in the 4FGL-DR2 catalog remains unassociated or unidentified (for brevity, we will label both unassociated and unidentified sources as UNC, unclassified, in what follows).

Given the rapid growth in size and complexity of astronomical datasets, machine learning algorithms have become increasingly powerful tools, in particular for object classification tasks. Automated schemes to classify gamma-ray sources based on machine learning techniques are instrumental to provide a list of particularly interesting candidate target sources for follow-up observations, as well as for enlarging the number of detected objects for population studies. Past works have mainly focused on using source features collected in gamma-ray catalogs only. Machine learning has been applied to investigate the nature of the UNC sources in current and past FermiLAT catalogs, see e.g. [5, 6], or to predict the subclass of AGN of uncertain type, see e.g. [7]. The studies thus far typically use standard machine learning algorithms, such as classification trees, logistic regression, or random forest classifiers, and are based on the features provided by the FermiLAT catalogs, or simple additional ones, such as hardness ratios, constructed from the energy and time dependent gamma-ray fluxes, see e.g. [5]. Such an approach requires a careful choice of the 

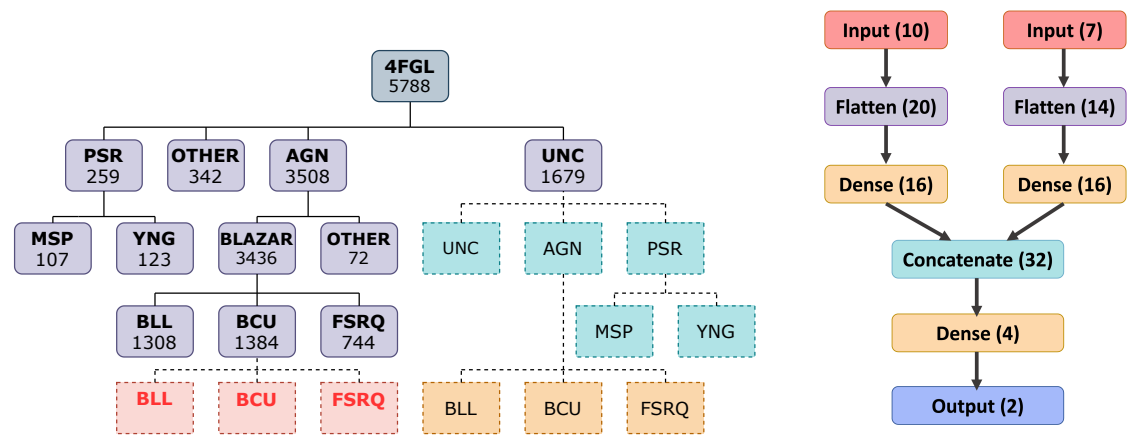

Figure 1: Left: Composition of Fermi-LAT 4FGL-DR2 catalog. Boxes connected by solid lines decipt the source classes and the number of sources in each class as reported in the catalog, while dashed lines indicate the further classification steps explored here using deep learning. See text and [1] for acronym definitions. Right: Sketch of the dense neural network architecture for the classification of Fermi-LAT sources. Each box illustrates one layer, stating the type of layer and the output shape in brackets.

features to consider, introducing ambiguities and potential biases e.g. due to existing knowledge of the source sample. Moreover, the features might not represent all the information encoded in the spectral data, and the physical interpretation of the importance of some features for the classifier, for example errors in the flux measurement, may be difficult. In addition, up to now machine learning gamma-ray source classification techniques do not provide reliable error estimates for the assignment of sources.

We propose a novel approach to classify gamma-ray sources of uncertain type with deep learning techniques. The central idea of our classification procedure is to directly use the gammaray energy spectra and time series, rather than a selection of derived features. We employ a fully connected deep neural network and a recurrent neural network trained directly on the energy and time dependent fluxes presented in the Fermi-LAT catalog. Bayesian inference methods are also explored to gain more information about the accuracy and the robustness of the neural networks predictions.

\section{Dataset and Methods}

The reference dataset is the 4FGL-DR2 catalog ${ }^{1}$, in which each source is described by measurements as well as human-crafted features (a total of 74) designed to represent the main source properties. We here use the gamma-ray flux values in the seven energy bands as reported in the 4FGL-DR2 (entry called Flux_Band, referred to as energy band/spectra/series data in what follows). As for the time spectra, we select the ten one-year light curves integrated over the full energy range (Flux_History, referred to as time spectra/series in what follows).

We use deep neural networks, i.e. neural networks composed of several layers, for the classification of gamma-ray sources. Three deep neural network architectures are explored: a fully connected network, also called dense neural network (DNN), a recurrent neural network (RNN), and a Bayesian neural network (BNN). RNNs are designed for analysing sequential data or timeseries data, and can process variable length sequences of inputs. Bayesian neural networks assign

\footnotetext{
${ }^{1}$ Publicly available at https://fermi.gsfc.nasa.gov/ssc/data/access/lat/10yr_catalog/
} 
an explicit prior to the distribution of network parameters, allowing us to assign uncertainties to the network predictions [8]. The resulting uncertainties depend on the statistical and systematic uncertainties of the dataset as well as the convergence of the network training. We concentrate on three binary-type classification tasks. We first perform with the DNN and RNN interclass binarytype classification, by predicting if UNC are AGN or PSR, and then PSR intraclass binary-type classification separating YNG vs. MSP (see cyan boxes in Fig.1, left panel and [9]). We then explore within the DNN and the BNN another binary-type classification tasks, predicting if blazars of uncertain type (BCU) are BLL or FSRQ (red boxes in Fig.1, left panel and [10, 11]). A visualisation of the DNN architecture is shown in Fig. 1 (right panel) for illustration. The DNN consist of two branches, one for the energy spectrum and one for the time series, which are eventually merged into a fully connected network for classification. In the dense layers we use $\operatorname{ReLU}(x)=\max (0, x)$ as activation function, except for the final layer where the softmax activation converts our output into a probability distribution. Both our neural network architectures are very flexible and additional features, apart from the energy and time spectra, can easily be included in the classification algorithms by appending a third branch to the architecture, which is merged for classification with the other two branches. We have used a ten-fold cross-validation procedure to optimise the hyperparameters of our networks (the number of layers in each branch, nodes in these layers, layers in the final classification part, the number of nodes within these layers), see [9] for more details. Just like the DNN, also the default RNN architecture consist of two branches, one for the energy and one for the time series. The main difference to the DNN architecture is that the dense layers are now substituted with recurrent layers. We find good classification results for three recurrent layers per branch with eight units each. After concatenating the two branches, 16 features are the input to the classification part of the network. This part is the same for the RNN as for the DNN. The recurrent layers used for the serial data are SIMPLERNN layers from TENSORFLOw/KeRAS ${ }^{2}$ which use a hyperbolic tangent activation function. For simplicity, the BNN is implemented considering the energy spectrum data only. The architecture for the BNN is modified such as each layer is substitute with a Bayesian layer using DenseFlipout layers from TensorFlow Probability. A similar hyperparameter search is performed to optimize the performance of the BNN.

As a first step in data preparation, we select sources from the 4FGL-DR2 catalog falling within the broad classes of AGN and PSR, and of the two main types of blazars, FSRQ and BLL. The PSR class includes all sources that are identified as or associated with pulsars (CLASS1=PSR, psr). As for the AGN broad class, we include all sources that are identified as or associated with any type of AGN, including subclasses of blazars as the Flat Spectrum Radio Quasars (FSRQ), BL Lacs (BLL), blazars of unknown type (BCU), and other more rare AGN types ${ }^{3}$. We will refer to identified/associated sources as labelled sources. The UNC sources are instead those without any identification or association, report with a blank space in their class attribute in the 4FGL-DR2 catalog. Since we are also interested in the classification of pulsars as YNG or MSP, we use the public list of LAT-detected gamma-ray pulsars ${ }^{4}$ to build a cross-match between this list and the

${ }^{2}$ https://www. tensorflow.org/, https://keras.io

3i.e. with CLASS1=FSRQ, fsrq, BLL, bll, BCU, bcu, CSS, cSs, RDG, rdg, NLSY1, nlsy1, agn, ssrq, sey, see $[1,2]$ for more details on the AGN subclass definition.

${ }^{4}$ https://confluence.slac.stanford.edu/display/GLAMCOG/Public+List+of+LAT-Detected+ Gamma-Ray+Pulsars 
4FGL-DR2 catalog. We shift and re-scale each data input such that the mean over the training set is zero and the standard deviation is one. The training of our neural networks is characterised by the choice of the optimiser, the loss function, the initial learning rate and the number of training updates in terms of batch size and number of epochs. We use the Adam optimiser [12] to minimise the categorical cross-entropy loss function [9]. We find that efficient training is achieved for the DNN with a batch size of 64 and an initial learning rate of 0.01 . For the RNN a batch size of 32 and an initial learning rate of 0.001 performs best. We train the networks for 50 and 150 epochs for the task of AGN vs. PSR and MSP vs. YNG classification. As for the BNN, a batch size of 32, an initial learning rate of $10^{-3}$ and 250 epochs are found to give the best performances in terms of accuracy and AUC. We train our networks on $70 \%$ of the labelled sources and evaluate on the remaining $30 \%$. The test split is performed such that the relative amount of the two classes is the same in the training and test sets. The performance measures and uncertainties correspond to the mean values and the standard deviations of ten training runs. For each run a new network is initialised and the training and test split is performed randomly. The uncertainties thus combine those from different network initialisations and from the selection of the training data. Due to the large imbalance of some classes in our dataset (see Fig.1), the measures used to quantify the performance of the classifier need to be selected with some care. We have considered an extensive list of complementary measures [9], but will report here only the global accuracy (ratio of correctly classified sources over the total number of sources) and the Area under the Receiver Operating Characteristic curve (AUC) for brevity. Moreover, we have also explored data augmentation techniques to balance and augment the small dataset we are dealing with. We report here results for the BNN adopting the standardly used SMOTE algorithm [13], see [9, 11] for extended discussion and results.

\section{Results with the dense and recurrent neural networks}

For the AGN vs. PSR classification with the benchmark DNN (RNN) we obtain an accuracy of $97.60 \pm 0.20(97.67 \pm 0.24)$ and $\mathrm{AUC}=97.99 \pm 0.75 \%(97.40 \pm 0.89 \%)$. Using only the energy spectrum data and no additional information about the time dependence (only right branch in Fig.1), we reach a similar performance, while using only the time series, we observe a decrease in performance (accuracy of $95.46 \pm 0.33 \%$ ). We conclude that the essential information for the AGN vs. PSR classification is contained in the energy spectrum integrated over time, and the time series data does not provide significant additional information. The same is observed for the RNN architecture. The performance of our classifiers have been tested on numerous architecture variations, as well as on a cross-match set of sources between an older Fermi-LAT catalog and the 4FGL-DR2 catalogs, see [9]. We have also explored different data augmentation techniques, finding that no benefit is achieved for the DNN and RNN classifiers, as these effects can be compensated for by a change in the decision threshold. The classification of MSP vs. YNG pulsars is significantly more difficult, given the very small data set, see Fig.1. In addition to the energy spectrum and time series information, we include the latitude of the pulsar in Galactic coordinates (GLAT) here, since it is a distinguishing feature that can be directly measured, as used also in $[5,6]$. Using the DNN (RNN) on the energy spectrum and the GLAT, we obtain an accuracy for MSP vs. YNG classification of $87.10 \pm 3.86 \%(85.51 \pm 3.37 \%)$ and $\mathrm{AUC}=92.84 \pm 3.41 \%(92.61 \pm 2.83 \%)$. As for the classification of blazars of uncertain type into FSRQ and BLL using the DNN, we obtain an accuracy of $88.77 \pm 1.00 \%$ and $\mathrm{AUC}=90.41 \pm 0.61 \%$ using the energy and time spectra, similar 
to previous works [7]. We also explored [10] the performance of the DNN, RNN with different data augmentation techniques and adding additional features, for example the synchrotron peak frequency as given in [14], finding slight improvements.

The DNN (RNN) classifier predicts that among the 1679 UNC sources in the 4FGL-DR2 catalog, 1050 (1076) are candidates for AGN (62\%/64\%), 78 (57) for PSR (4.6\%/3.4\%), while 551 (546) remain unclassified. These 551 sources are mostly concentrated along the Galactic plane, where the bright Galactic emission likely prevents a precise measurement of their energy and time spectra. The percentage of AGN and PSR candidates is in line with similar estimates in previous publications [5], and in fair agreement with the distribution among labelled sources in the 4FGLDR2 catalog (61\% AGN and 4.5\% PSR). The predicted labels for UNC sources obtained with the two architectures are found to be consistent. As for the MSP vs. YNG pulsars classification, we identified a list of 11 promising MSP candidates identified by both our DNN GLAT and RNN GLAT architectures, detailed in Table 7 in [9]. Finally, using the benchmark DNN architecture, we identify 853 BLL and 531 FSRQ candidates among the BCU.

The latitude distribution of AGN, YNG and MSP candidates obtained with the DNN classifier, together with the UNC sources in the 4FGL-DR2 catalog, is presented in Fig. 2, and is found to be compatible with the expected distribution inferred from labelled sources, with PSR candidates clustered along the Galactic plane, and AGN candidates distributed more isotropically. Although our DNN and RNN architectures use only the energy and time spectra for training, testing, and in order to provide a prediction for the UNC sources, the distribution of relevant features can be analysed afterwards. In Fig. 3 (left panel) we show a variability-curvature contour plot for the labelled sources in the 4FGL-DR2 catalog, obtained by extracting the variability index (Variability_index) and the curvature significance for the log parabola spectral fit (LP_SignCurv). ${ }^{5}$ Even though the DNN is not trained to separate sources based on these features (as done instead when using feature selection [5, 6]), the distribution of candidate sources (AGN: red circles, PSR: blue squares) follows the expected clustering in the variability-curvature plane (red and blue contours), confirming that the neural networks extract the relevant features from the energy and time spectra. Similar results are obtained for the RNN. Comparable conclusions are drawn for the results of the BCU classification: the distribution of relevant features, such as the power law photon index or the variability index for the BLL and FSRQ candidates nicely follows the one of labelled sources in the 4FGL-DR2[10].

\section{Results with the Bayesian neural network}

To further estimate the accuracy and the robustness of the neural network predictions, we explore Bayesian inference methods. We start with the classification of blazars of uncertain type into BLL and FSRQ, as this dataset is fairly balanced wrt. for example the AGN vs. PSR one. Results for the training and testing of the BNN on the 4FGL-DR2 labelled sources are presented. The distribution of the classification uncertainties obtained for the FSRQ class is shown in Fig.3 (right panel), where we show the standard deviation against the mean FSRQ score for 500 draws of the BNN weights. The scatter points correspond to individual sources, the histograms to the mean values within the bins. We tested three different amounts of augmentation using SMOTE: no augmentation at all (No aug), augmentation of the under represented class to even the number

\footnotetext{
${ }^{5}$ In the variability-curvature plane AGN and PSR are known to cluster in different regions, as PSR tend to be non-variable on long time scales and have spectra exhibiting significant curvature (see e.g. [1]).
} 

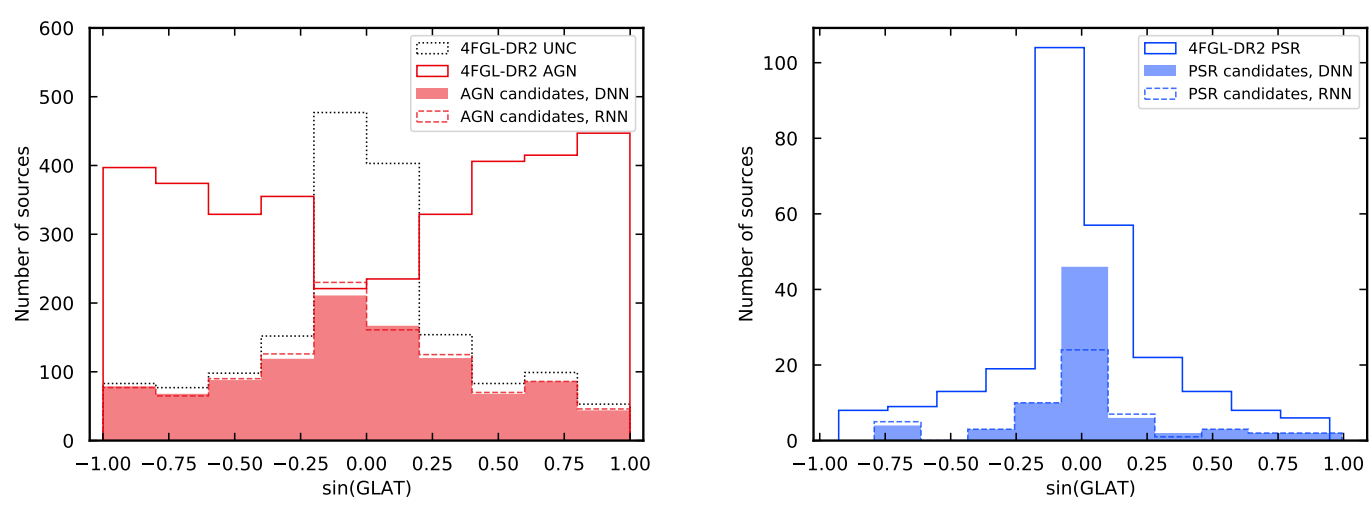

Figure 2: Galactic latitude distribution of AGN (left panel) and PSR (right panel) of 4FGL-DR2 sources. In each panel, the distribution of AGN/PSR labelled sources in the 4FGL-DR2 is reported together with the distribution of source candidates as derived by two neural network architectures. The distribution of UNC sources is also shown in the left panel for comparison.
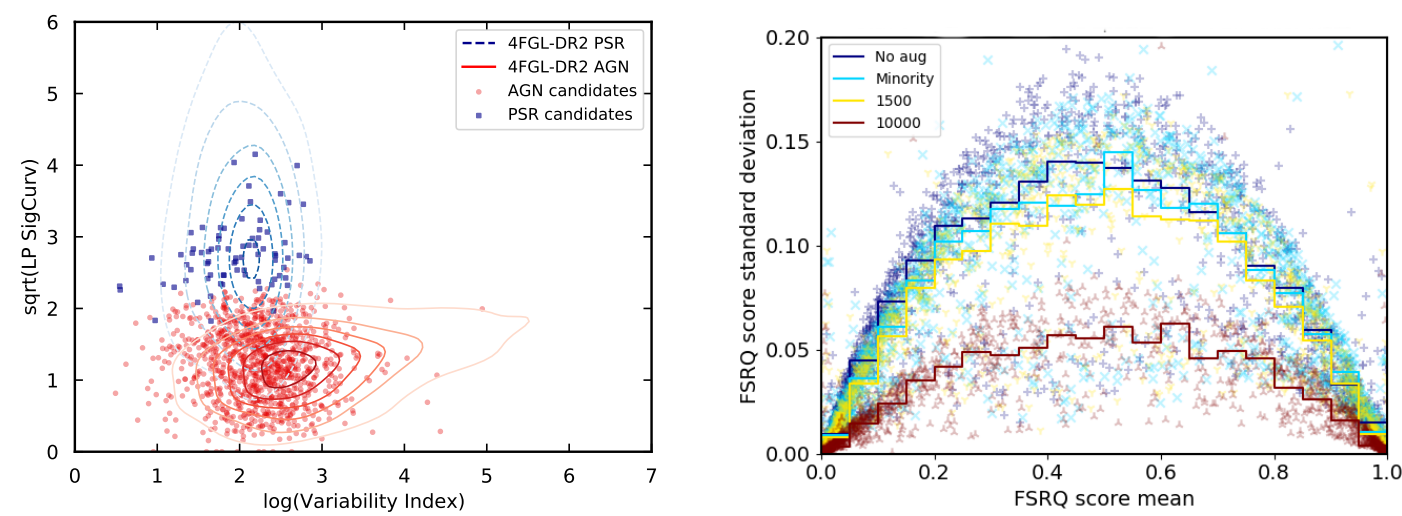

Figure 3: Left: Curvature-Variability plot (Sqrt(LP SignCurv) vs. Log(Variability Index)) for labelled AGN (red contours) and PSR (blue dashed contours) sources in the 4FGL-DR2 catalog. The distribution of candidate AGN (red circles) and PSR (blue squared) obtained from the DNN is superimposed. Right: distribution of the classification uncertainties for the FSRQ class with the BNN and different data augmentation setup.

of sources with the other class (Minority) and fixing the number of sources per class, such that for both classes augmented sources exist $(1500,10000)$. Adding augmented data reduces the statistical uncertainty of the dataset. The result is a significantly reduced uncertainty on the prediction, which is now limited by systematic uncertainties in the dataset. For just augmenting the minority class and small amounts of data augmentation, the effect is rather small. The BNN predictions for the BCU will be detailed in [11].

\section{Conclusions}

We demonstrated that deep neural networks based on energy and time spectra provide powerful classifiers to separate AGN from PSR, YNG from MSP, and blazars of uncertain type into FSRQ and BLL, with a performance that is comparable with previous feature-based analyses presented in 
the literature. We explored Bayesian neural networks, and present preliminary results in the context of the classification of blazars of uncertain type. Results presented in this contribution are a subset of the work detailed in [9-11]. We plan to use the predicted classification with uncertainties to complement population studies of extragalactic and Galactic sources, and to extend our data inputs to include multiwavelength data for the energy and time spectra coming from different observatories.

\section{References}

[1] Fermi-LAT Collaboration, S. Abdollahi et al., Fermi Large Area Telescope Fourth Source Catalog, ApJS 247 (2020), no. 133, [1902 . 10045].

[2] Fermi-LAT Collaboration, J. Ballet, T. Burnett, S. Digel, and B. Lott, Fermi Large Area Telescope Fourth Source Catalog Data Release 2, 5, 2020.

[3] P. Padovani et al., Active galactic nuclei: what's in a name?, Astron. Astrophys. Rev. 25 (2017), no. 12, [1707.07134].

[4] A. A. Abdo, M. Ackermann, M. Ajello, W. B. Atwood, M. Axelsson, et al., A Population of Gamma-Ray Millisecond Pulsars Seen with the Fermi Large Area Telescope, Science 325 (Aug., 2009) 848.

[5] P. M. Saz Parkinson, H. Xu, P. L. H. Yu, D. Salvetti, M. Marelli, et al., Classification and ranking offermilat gamma-ray sources from the 3 fgl catalog using machine learning techniques, ApJ 820 (Mar, 2016) 8.

[6] S. Luo, A. P. Leung, C. Y. Hui, and K. L. Li, An investigation on the factors affecting machine learning classifications in gamma-ray astronomy, MNRAS 492 (Jan, 2020) 5377-5390.

[7] M. Kovačević, G. Chiaro, S. Cutini, and G. Tosti, Classification of blazar candidates of uncertain type from the Fermi LAT 8-yr source catalogue with an artificial neural network, MNRAS 493 (2020), no. 2 1926-1935, [2002 . 10256].

[8] C. M. Bishop, Bayesian Neural Networks, J. Braz. Comp. Soc. 4 (1) (1997).

[9] T. Finke, M. Krämer, and S. Manconi, Classification of Fermi-LAT sources with deep learning using energy and time spectra, 2012.05251.

[10] F. Keil, Classification of Astrophysical gamma-ray sources with Machine Learning, RWTH Bachelor thesis (2021).

[11] A. Butter, T. Finke, F. Keil, M. Krämer, and S. Manconi In preparation (2021).

[12] D. P. Kingma and J. Ba, "Adam: A method for stochastic optimization." Conference paper at the 3rd International Conference for Learning Representations, San Diego, 2015, 2017.

[13] N. V. Chawla, K. W. Bowyer, L. O. Hall, and W. P. Kegelmeyer, Smote: synthetic minority over-sampling technique, Journal of artificial intelligence research 16 (2002) 321-357.

[14] Fermi-LAT Collaboration, M. Ajello et al., The Fourth Catalog of Active Galactic Nuclei Detected by the Fermi Large Area Telescope, Astrophys. J. 892 (2020) 105, [1905. 10771]. 